\title{
Observation of Faraday Waves in a Bose-Einstein Condensate
}

\author{
P. Engels* and C. Atherton \\ Washington State University, Department of Physics and Astronomy, Pullman, WA, 99164 \\ M. A. Hoefer \\ National Institute of Standards and Technology, Boulder, Colorado 8030年
}

(Dated: August 9, 2018)

\begin{abstract}
Faraday waves in a cigar-shaped Bose-Einstein condensate are created. It is shown that periodically modulating the transverse confinement, and thus the nonlinear interactions in the BEC, excites small amplitude longitudinal oscillations through a parametric resonance. It is also demonstrated that even without the presence of a continuous drive, an initial transverse breathing mode excitation of the condensate leads to spontaneous pattern formation in the longitudinal direction. Finally, the effects of strongly driving the transverse breathing mode with large amplitude are investigated. In this case, impact-oscillator behavior and intriguing nonlinear dynamics, including the gradual emergence of multiple longitudinal modes, are observed.

PACS numbers: 03.75.Kk,67.90.+z,05.45.-a,47.54.-r
\end{abstract}

In 1831, Faraday studied the behavior of liquids that are contained in a vessel subjected to oscillatory vertical motion [1]. He found that fluids including alcohol, white of egg, ink and milk produce regular striations on their surface. These striations oscillate at half the driving frequency and are termed Faraday waves. They are considered to be an important discovery. Since then, the more general topic of pattern formation in driven systems has been met with great interest, and patterns have been observed in hydrodynamic systems, nonlinear optics, oscillatory chemical reactions, and biological media [2] .

In this paper we study pattern formation by modulating the nonlinearity in a Bose-Einstein condensate (BEC). Nonlinear dynamics arise from the interatomic interactions in this ultracold gas. In the past the observation of interesting phenomena has motivated researchers to propose and implement various techniques to manipulate the nonlinearity. Such control has been accomplished for example by exploiting Feshbach resonances [3]. In our experiment we investigate an alternative technique, namely periodically modulating the nonlinearity by changing the radial confinement of an elongated, cigar-shaped BEC held in a magnetic trap. The radial modulation leads to a periodic change of the density of the cloud in time, which is equivalent to a change of the nonlinear interactions and the speed of sound. This can, in turn, lead to the parametric excitation of longitudinal sound-like waves in the direction of weak confinement. This process is analogous to Faraday's experiment where the vertical motion of the vessel produced patterns that were laterally spread out.

It has been shown theoretically that for a BEC, a Faraday type modulation scheme in the case of small driving frequencies leads one to the same type of analysis as would the direct modulation of the interatomic interaction, e.g., by a Feshbach resonance [4, 5]. In both cases, the dynamics are governed by a Mathieu equation that is typical for parametrically driven systems. Floquet analysis reveals that a series of resonances exist, consisting of a main resonance at half the driving frequency, and higher resonance tongues at integer multiples of half the driving frequency [4].

In our experiment we exploit this transverse modulation scheme for three different applications. First, we apply a relatively weak continuous modulation, demonstrate the emergence of longitudinal Faraday waves, and study their behavior as a function of the excitation frequency. Second, we investigate longitudinal patterns that emerge as a consequence of an initial transverse breathing mode excitation without the presence of a continuous drive. This has important consequences in the context of damped BEC oscillations and has been studied theoretically in [6]. Since the first experiments with BECs, the study of collective excitations has been a central theme 7, 8]. The transverse breathing mode, which we exploit in our experiments, plays a prominent role: Chevy et al. 9] showed that this mode exhibits unusual properties, namely an extremely high quality factor and a frequency nearly independent of temperature. Finally, in a third set of measurements we study the situation of a relatively strong modulation, resonantly driving the transverse breathing mode. We show that the condensate responds as an impact oscillator, which leads to intriguing multimode dynamics.

The experiments were carried out in a newly constructed BEC machine that produces cigar-shaped condensates of ${ }^{87} \mathrm{Rb}$ atoms in the $\left|F=1, m_{F}=-1\right\rangle$ state. The typical atom number in the BEC is $5 \cdot 10^{5}$, and the atoms are evaporatively cooled until no thermal cloud surrounding the condensate is visible any more. The atoms are held in a cylindrically symmetric IoffePritchard type magnetic trap with the harmonic trapping frequencies of $\left\{\omega_{x y} /(2 \pi), \omega_{z} /(2 \pi)\right\}=\{160.5,7\} \mathrm{Hz}$. The weakly confined z-direction is oriented horizontally. 
For the experiments described below, the following collective mode frequencies are of particular importance: first, there exists a high-frequency transverse breathing mode. For our trap geometry, this mode has a frequency of $\omega_{\perp} /(2 \pi)=321 \mathrm{~Hz}[10,11]$, very close to the limit of vanishing axial confinement $2 \cdot \omega_{x y} /(2 \pi)$. The second set of modes in which we are interested here consists of axial modes which, for large quantum numbers, correspond to sound waves in the z-direction. The frequencies of this discrete set of modes can be approximately calculated as given in [12, 13].

In order to investigate the parametric driving process mentioned above, we first performed a set of "spectroscopy" experiments in which we continuously modulated the transverse trapping confinement at a fixed modulation frequency and observed the subsequent emergence of longitudinal Faraday waves. For each excitation frequency the modulation amplitude was adjusted such that the longitudinal patterns emerged typically at some point after 10 to 30 oscillations. On the breathing mode resonance, a trap modulation of $3.6 \%$ was used, while at many other frequencies trap modulations of up to $42.5 \%$ were chosen to obtain clearly visible patterns [14]. This range of modulation depths is similar to the range used in numerical simulations in [5]. Representative examples for the resulting patterns are shown in Fig. 1. All experimental images in this manuscript were taken by destructive in-trap imaging.

The average spacing of adjacent maxima in the resulting pattern is plotted against the driving frequency in Fig. 2. The data lie on a clear curve, with the exception of the points near a driving frequency of $160.5 \mathrm{~Hz}$, corresponding to the transverse dipole mode resonance (i.e. transverse slosh motion) [10]. However, inspection of our experimental images reveals that, at this frequency, we also excite the transverse breathing mode at $321 \mathrm{~Hz}$. Excitation of the breathing mode is a very effective way of creating longitudinal patterns. Therefore the patterns obtained at $160.5 \mathrm{~Hz}$ are actually the same as those produced at $321 \mathrm{~Hz}$. In order to rationalize the data, we first note that parametric excitation with a certain driving frequency excites oscillations predominantly at half the driving frequency, the main resonance also observed in Faraday's experiments. The dispersion relation of longitudinal collective modes that become sound-like for high quantum numbers is given in [12, 13] and is used to calculate the expected spacing between density maxima. The resulting spacings are plotted as the step-like curve in Fig. 2 and are in excellent agreement with our experimental data, corroborating the assumption and theory of a parametric driving process.

In a second set of experiments we show that the longitudinal modes are driven by the transverse breathing motion even without the presence of a continuous external drive. In particular, this disproves the influence of any tiny residual axial trap modulation that has existed

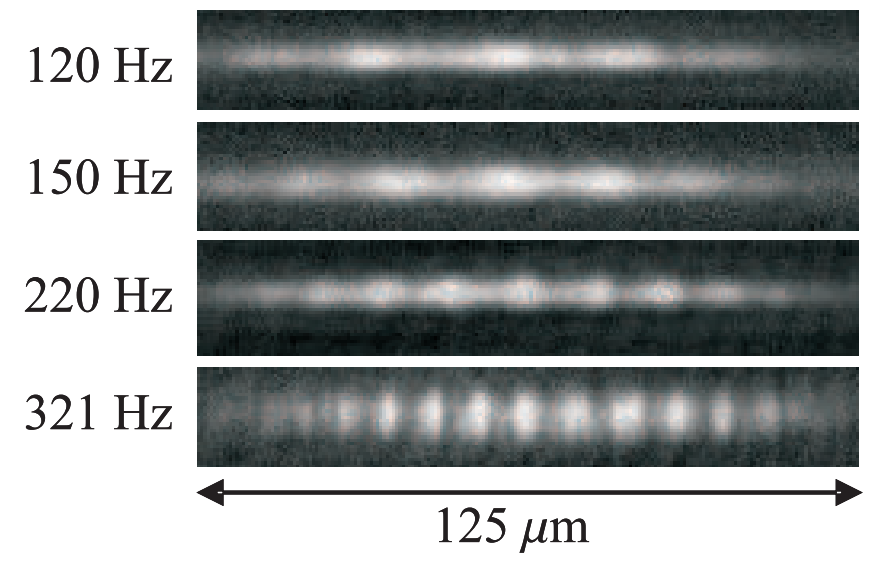

FIG. 1: In-trap absorption images of Faraday waves in a BEC. Frequency labels for each image represent the driving frequency at which the transverse trap confinement is modulated.

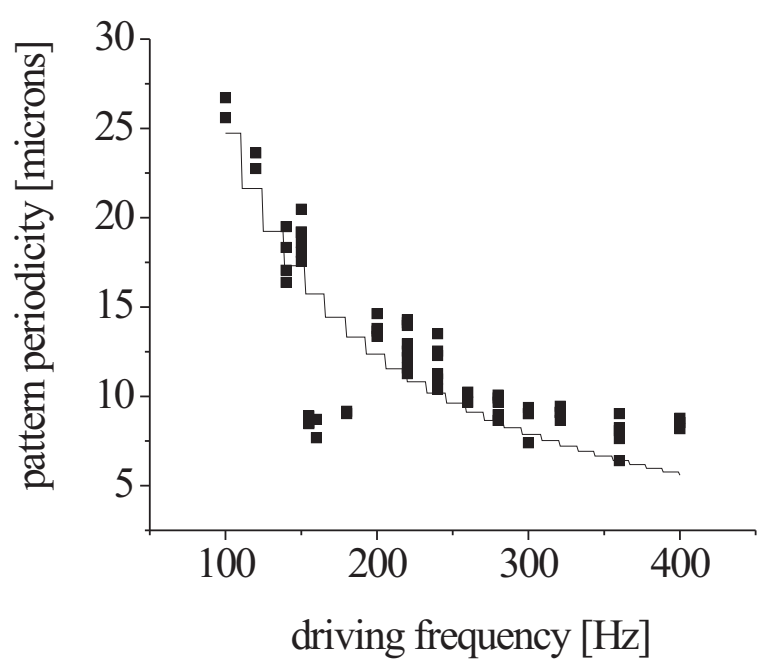

FIG. 2: Average spacing of adjacent maxima of the longitudinal patterns plotted versus the transverse driving frequency. Points are experimental data, while the line shows the theoretical values calculated for the longitudinal modes closest to half the driving frequency.

during the presence of our continuous drive due to experimental imperfections [14]. For this, we excited the transverse breathing mode at $321 \mathrm{~Hz}$ by driving the transverse trap confinement for a few cycles, and then let the condensate evolve without the presence of the drive. For a gentle excitation, we do not see longitudinal patterns immediately after the end of the drive, but can observe them emerge at later times. A weaker excitation delays the emergence of longitudinal modes out to later times, while in the case of strong excitations the patterns can emerge within the first three cycles. In order to follow the evolution, we quantify the presence of longitudinal 


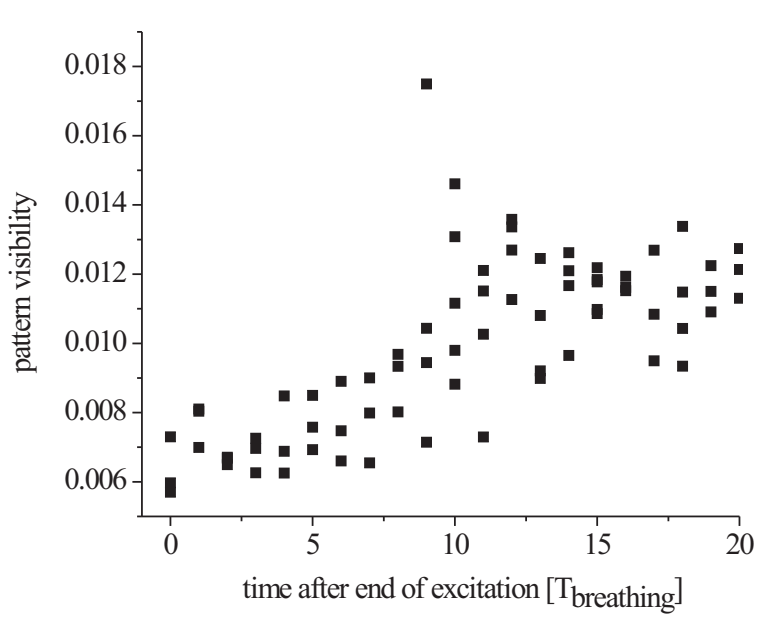

FIG. 3: Onset of Faraday wave formation after driving the transverse breathing mode for only two cycles. Pattern visibility is quantified as described in [15].

patterns as described in [15]. Fig. 3 shows the evolution after the end of a moderate excitation. For this data, we excited the condensate for only two cycles, varying the transverse trap frequency by $9 \%$ with a modulation frequency of $321 \mathrm{~Hz}$ during the modulation. Immediately after the excitation, the obtained images showed no longitudinal waves, and our pattern visibility measure, plotted in the figure, is initially picking up high frequency noise along the longitudinal axis. A main contribution to this noise is the imaging noise of our detection system. Weak pattern formation is observed starting at five periods after the end of the modulation, and strong longitudinal patterns then appear after about nine periods. A similar behavior is known for example from parametric amplifiers in optics: if no input signal amplitude is present, a signal emerging from noise (or zero point energy) can form if the amplification is large enough. This behavior is also found by our numerical simulations based on the Gross-Pitaevskii equation. In simulations with similar parameters as in the experiment, no pattern formation is observed for several breathing mode periods. From the onset of pattern formation, it takes just three periods for patterns to grow to their full strength. We find that the onset time of pattern formation in the simulations is earlier when more noise is added to the initial relaxed wave function.

This experiment is closely related to the theoretical situation described in [6], where a single and sudden jump in the transverse trap frequency was used to excite the breathing mode, instead of a sinusoidal trap frequency modulation. We have used a trap jumping excitation in the case of very elongated BECs to demonstrate a second observation about the onset of longitudinal patterns, namely the fact that these patterns can start to

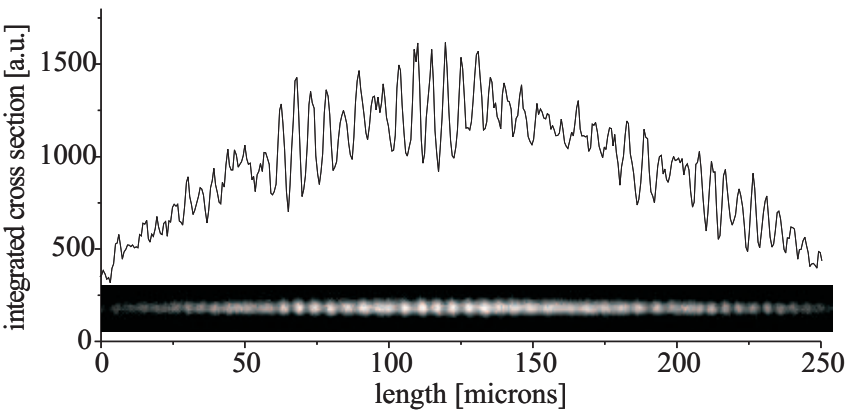

FIG. 4: Pattern domains observed in a very elongated BEC. The graph shows an integrated cross section of the BEC displayed in the inset.

emerge in spatially localized domains, rather than uniformly across the whole cloud. To show this effect, we produced condensates in a very elongated cigar-shaped trap with trapping frequencies of $\left\{\omega_{x y} /(2 \pi), \omega_{z} /(2 \pi)\right\}=$ $\{286.1,2.8\} \mathrm{Hz}$. We temporarily jumped to a different trap of $\left\{\omega_{x y} /(2 \pi), \omega_{z} /(2 \pi)\right\}=\{88.4,5.1\} \mathrm{Hz}$ for the duration of $1.3 \mathrm{~ms}$, then jumped back to the first trap and let the cloud evolve. After about $10 \mathrm{~ms}$, we observed BECs in which a perfectly periodic density modulation was stretching over almost the entire cloud; but it is also not uncommon to observe patterns in several separate domains, as shown in Fig. 4. Considering that the speed of sound in the elongated cloud is only about $2 \mathrm{~mm} / \mathrm{s}$ [13, 16], it is plausible that over the time scale of this experiment different regions of the $\mathrm{BEC}$ can get independently excited and evolve into longitudinal patterns independently from each other.

In the experiments described so far, our parametric excitation has led to longitudinal modes oscillating at half the frequency of the parametric driving frequency. In the case of strong parametric amplification, it is theoretically expected that modes at other frequencies (higher resonance tongues), in particular modes at the driving frequency, can be excited, too [4]. This motivated our third set of experiments in which we started again with a condensate in a trap with trap frequencies of $\left\{\omega_{x y} /(2 \pi), \omega_{z} /(2 \pi)\right\}=\{160.5,7\} \mathrm{Hz}$. We then continuously modulated our transverse trap frequency by about $19 \%$ with a modulation frequency of $321 \mathrm{~Hz}$. The resulting breathing motion is seen in Fig. 5 where we plot the Thomas-Fermi radius of the cloud in the transverse direction versus time. The graph clearly shows that the cloud, upon strong excitation, gradually starts behaving as an impact oscillator, i.e., as an oscillator bouncing off a stiff wall during each period. A classical impact oscillator, realized for example by a ball bouncing off a stiff surface, is a paradigm for nonlinear dynamics, nonlinear resonances and chaotic behavior. In the present case, the role of the stiff wall was played by the strong mean-field repulsion during the slim phase of the oscillations when the BEC is strongly compressed in the transverse direction. Theoret- 


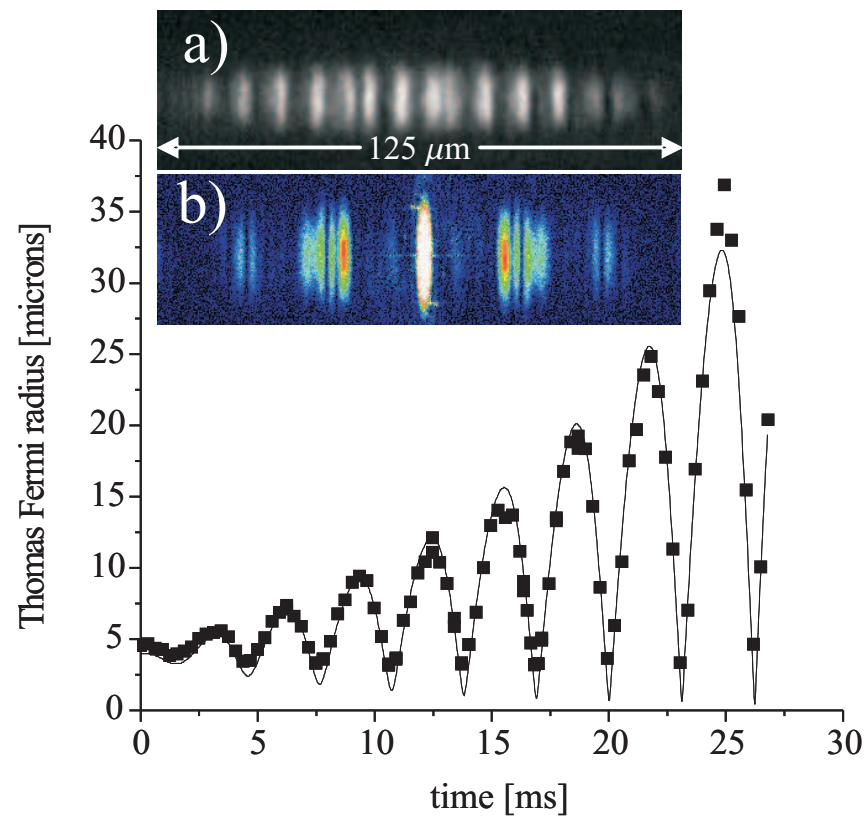

FIG. 5: Impact oscillator behavior. Plot of BEC radius in the tight direction vs. time when continuously driving the transverse breathing mode. Points show experimental data, while the curve corresponds to numerical simulation. Inset: (a) Image of BEC after 5.2 driving periods, i.e. $16.2 \mathrm{~ms}$. (b) Fourier transform of the image in (a).

ically, the instability of the breathing mode upon strong driving and the impact oscillator behavior have been analyzed in [17]. The behavior of the cloud radius in the transverse direction was also reproduced in our numerical simulation of the azimuthally symmetric 3D GrossPitaevskii equation, displayed as the solid line in Fig. 5. However, the numerics, when starting with a thoroughly relaxed wave function in the initial trap, show a sign of longitudinal pattern formation only after $18 \mathrm{~ms}$, while in the experiment, longitudinal patterns clearly formed already during the third period (9 ms). This, again, hints at the importance of initial noise in the condensate that seeds the parametric amplification. In the experiment, the patterns start out similar to those displayed in Fig. 1 for the case of a weak drive at $321 \mathrm{~Hz}$. But, upon the action of the strong drive, they quickly evolve into more complicated patterns, involving the excitation of several other modes. The inset of Fig. 5 shows an image taken after 5.2 driving periods (a), together with the Fourier transform (b). The Fourier spectrum reveals that several modes corresponding to the first resonance tongue of longitudinal modes with nearly half the driving frequency are excited. In addition, modes at twice the distance from the central Fourier peak are visible. Those modes belong to the second resonance tongue of the main resonance.

In conclusion, we have experimentally observed the effects of parametric resonances in a BEC. The observed resonances lead to Faraday waves along the long BEC axis. These results advance the understanding of collective mode behavior in a condensate, which is one of the key tools to study BEC dynamics. In addition, we have shown that strongly driving the transverse breathing mode leads to an instability whereupon the mode amplitude increases exponentially, accompanied by the strong excitation of multiple sound-like modes.

* Electronic address: engels@wsu.edu

$\dagger$ Contribution of the U.S. Government. Not subject to copyright.

[1] The study is contained in the appendix of the paper M. Faraday, Philos. Trans. R. Soc. London 121, 299 (1831).

[2] For a comprehensive review, see M. C. Cross and P. C. Hohenberg, Rev. Mod. Phys. 65, 8511112 (1993).

[3] S. Inouye, M. R. Andrews, J. Stenger, H.-J. Miesner, D. M. Stamper-Kurn, and W. Ketterle, Nature (London) 392, 151 (1998).

[4] K. Staliunas, S. Longhi, and G. J. de Valcárcel, Phys. Rev. Lett. 89, 210406 (2002).

[5] K. Staliunas, S. Longhi, and G. J. de Valcárcel, Phys. Rev. A 70, 011601(R) (2004).

[6] Yu. Kagan and L. A. Maksimov, Phys. Rev. A 64, 053610 (2001).

[7] D. S. Jin, J. R. Ensher, M. R. Matthews, C. E. Wieman, and E. A. Cornell, Phys. Rev. Lett. 77, 420 (1996).

[8] M.-O. Mewes, M. R. Andrews, N. J. van Druten, D. M. Kurn, D. S. Durfee, C. G. Townsend, and W. Ketterle, Phys. Rev. Lett. 77, 988 (1996).

[9] F. Chevy, V. Bretin, P. Rosenbusch, K. W. Madison, and J. Dalibard, Phys. Rev. Lett. 88, 250402 (2002).

[10] S. Stringari, Phys. Rev. Lett. 77, 2360 (1996).

[11] V. M. Pérez-García, H. Michinel, J. I. Cirac, M. Lewenstein, and P. Zoller, Phys. Rev. Lett. 77, 5320 (1996).

[12] M. Fliesser, A. Csordás, P. Szépfalusy, and R. Graham, Phys. Rev. A 56, R2533 (1997).

[13] S. Stringari, Phys. Rev. A 58, 2385 (1998).

[14] Sinusoidally modulating our bias field strength led to a modulation of the transverse confinement $\omega_{x y} /(2 \pi)$ between $155 \mathrm{~Hz}$ and $166.7 \mathrm{~Hz}$ in the case of a $3.6 \%$ modulation depth, and between $122 \mathrm{~Hz}$ and $303 \mathrm{~Hz}$ for our strongest modulation depth of $42.5 \%$. Even with our strongest transverse modulation, the longitudinal confinement was modulated by less than $0.6 \%$.

[15] We integrate the observed density along the tight direction, and determine the standard deviation of the resulting cross section from a Thomas-Fermi shape in the central region. We normalize the result by dividing by the number of atoms in the same region. The Thomas-Fermi shape is obtained by eliminating all but the low frequency Fourier components that correspond to the overall shape of the BEC. This procedure provides a good measure for the excitation of the longitudinal modes, which we have checked also by visual inspection of the data.

[16] E. Zaremba, Phys. Rev. A 57, 518 (1998).

[17] J. J. G. Ripoll and V. M. Pérez-García, Phys. Rev. A 59, 2220 (1999); J. J. García-Ripoll, V. M. Pérez-García, and P. Torres, Phys. Rev. Lett. 83, 1715 (1999). 